\title{
The Assessment of Quality of Life Using GOHAI among Edentulous Patients
}

\author{
Tine Martina Winarti, Norlela Yacob*, Wan Nor Syariza Wan Ali, Aida Ali \\ Department of Prosthodontics and Conservative Dentistry, Faculty of Dentistry, Universiti Sains Islam Malaysa (USIM), Kuala Lumpur, Malaysia
}

${ }^{*}$ Corresponding author: Norlela Yacob, Department of Prosthodontics and Conservative Dentistry, Faculty of Dentistry, Universiti Sains Islam Malaysia, 71800 Nilai, Negeri Sembilan, Malaysia; Tel: +60342891140; E-mail: drnolela@usim.edu.my

Received: January 23, 2019; Accepted: January 29, 2019; Published: January 31, 2019;

\begin{abstract}
Introduction: Edentulism may compromise the quality of life (QoL) of a patient. Geriatric patients who are satisfied with their complete dentures are usually satisfied with their daily life. This research was conducted to assess quality of life using Geriatric Oral Health Assessment Index (GOHAI) focusing on functional limitation, oro-facial pain, psychological impact and behavioural impact among edentulous patient attending USIM Polyclinic.
\end{abstract}

Materials and Methods: Thirty-two geriatric edentulous patient to be treated by third year students in polyclinic USIM were recruited. Patient completed the Malay version of GOHAI questionnaire.

Result: The data showed there were $62.5 \%$ male and $37.5 \%$ female. The mean age was $63.88 \pm 5.621$ year. $87.5 \%$ of the samples are wearing complete denture and $56.3 \%$ of them have been wearing denture for the past five years. The lowest mean score was $1.66( \pm 1.6)$ for trouble in biting and chewing. The highest mean for GOHAI was in medication used to relieve pain with the mean score of $4.56( \pm 0.8)$.

Conclusion: The QoL of geriatric patient was mostly affected by the oral function. However the least affected was seen in pain and discomfort. Despite wearing complete denture, denture replacement is essential to improve QoL.

Keyword: Edentulous and Quality of life, GOHAI, Geriatric Dentistry

\section{Introduction}

According to the Gerodontology society, the life expectancy of the elderly who need complete denture prosthesis has increased [1]. Edentulism is considered as an outcome of poor oral healthcare and may compromise the quality of life [2]. The physiological changes in edentulous arch with continuous resorption of the residual ridges leads to the need of complete denture replacements. The use of complete denture increases the quality of life of geriatric patient in term of oral function, reduce oro-facial pain, improve oro-facial appearance, and psychosocial impact [3]. Oral health is often assessed separately from patient's general health. Sometimes it perceived as a distinct entity from any chronic conditions of the elderly patient. Edentulism may compromise the quality of life (QoL) because patients will have difficulty eating proper food and this might lead to malnourish among the elderly. Edentulous patients need dentures to restore their oral function. Satisfaction of the denture influences psychological condition which is part of quality of life (QoL). Geriatric patient who are well satisfied with their daily lives, usually also satisfied with their complete denture [4].

There is a measure called Geriatric/General Oral Health Assessment Index (GOHAI) is an instrument consists of 12-item questionnaires and it is considered a gold standard used in measuring the oral health impact of the geriatric patients. It was developed by
Atchinson and Dolan [4] and has been validated over a few languages including Malay language [5] which is useful to measure oral-health related quality of life in Malaysia. From its name, GOHAI Index is usually used for the assessment of the quality of life in geriatric patient and not suitable to assess the overall oral health status because there was a weak correlation with the clinical measure of disease. Therefore, GOHAI cannot be used to conclude a diagnosis of dental disease because it will never assess the oral examination clinically and radiographically. However, indication for a referral or further thorough oral examination need can be obtained through GOHAI assessment as it will provide necessary information in symptoms as well as the limitation in psychosocial and functional. Therefore the aim of this paper was to assess the quality of life of the elderly population among the geriatric patients that received complete dentures from the undergraduate dental students of the Faculty of Dentistry, Islamic Science University of Malaysia (USIM) Polyclinic, Kuala Lumpur.

\section{Methods}

An approval was attained before starting the study by Board of Research Ethic of Islamic Science University of Malaysia (USIM). A total of 32 community dwelling participants were recruited among complete denture patients who will receive complete dentures from the undergraduate dental students of USIM. They must satisfy all the following inclusion criterias; Malaysians aged 60 years and above, 
complete denture wearers, subject with controlled or no underlying systemic diseases, and fluent in Malay language. The consents were obtained from participants after being informed about voluntary participation in this research. Then, self-administered questionnaire was distributed to collect information of participant's demographic and prosthetic experience.

The participants were interviewed by a single interviewer and were asked to estimate the frequency of problems in Malay Version of GOHAI questionnaire using a six point Likert scale rating (always [5], very often [4], often [3], sometimes [2], seldom [1] or never [0]). The 12 -item questionnaire was classified into four major domains which were the functional limitation, pain or discomfort, psychological and behavioural impact. The GOHAI score is determined by summing the final score of each of the 12 items ranges from 0 to 60 . The score for GOHAI item number 3, 5 and 7 were maintained while the rest were reversed in order to attain a positive oral health GOHAI score. The higher GOHAI score denotes better oral health status perceived by the participants themselves. If there were 3 or more missing items in the data, the data will be dropped out. Alternately, if no more than two missing items in a data, the score of the missing will be substituted with the mean. The mean GOHAI scores in relation with demographic variables were analyzed using Independent t-test and ANOVA. 0.05 was set as the level of significance. The data were analyzed using IBM SPSS Statistic version 19.

\section{Results}

The patients' age ranges from $60-76$ years with mean age of $63.88 \pm 5.6$ (as shown in (Table 1) below. Almost one-third of them were female and more than half received education up to secondary education level (11 years of formal education in school). Majority of them have been wearing a complete denture for more than 5 years (56.3\%). Most of the patients request for new dentures because they want to improve the chewing ability (50.0\%) and aesthetic reasons (40.6\%) over the other health and speech reasons. (Table 2)

Table 1. Distribution of patients according to sex.

\begin{tabular}{|l|c|c|c|}
\hline \multicolumn{1}{|c|}{ Gender } & Number & Mean & SD \\
\hline Male & 20 & 64.40 & 5.707 \\
\hline Female & 12 & 63.00 & 5.608 \\
\hline Total & 32 & 63.88 & 5.621 \\
\hline
\end{tabular}

The mean and median of the summary GOHAI scores was 40.1 ( \pm 7.4 , range $27-60$ ) and 39.0, respectively. Besides that, the skewness of the GOHAI was 0.75 . There was only one out of 32 subjects scored the maximum in this measure. About one-third of the participants (31.3\%) scored 41 and above. The mean of GOHAI scores in regard to each variable varies from $37.3( \pm 4.2)$ to $47.5( \pm 6.4)$. The lowest and highest mean GOHAI scores can be found in university level participants and reason of wearing denture for health purpose respectively (Table 2). Based on the data exhibited in Table 2, there is no significant association between gender, age, level of education, history of wearing denture and reason of wearing denture with the oral health related quality of life (OHRQoL). The used of medication to relieve pain in pain and discomfort domain was recorded as the highest mean for GOHAI item with 4.56 (SD 0.8) followed by 4.31 (SD 1.1) for sensitive to hot, cold or sweet foods and 4.31 (SD 1.3) for limit contact with people. The lowest mean was 1.66 (SD 1.6) in trouble biting and chewing. Meanwhile, Table 3 shows the percent of participants responding 'sometimes,' 'often,' 'very often,' or 'always' to each of the GOHAI items. The percentage of subjects responding positively to each item ranged from $16 \%$ to $80 \%$, with ten out of 12 items being reported by $20 \%$ or more and six of 12 reported by $33.3 \%$ or more (Table 3).

Table 2. Characteristics of subjects.

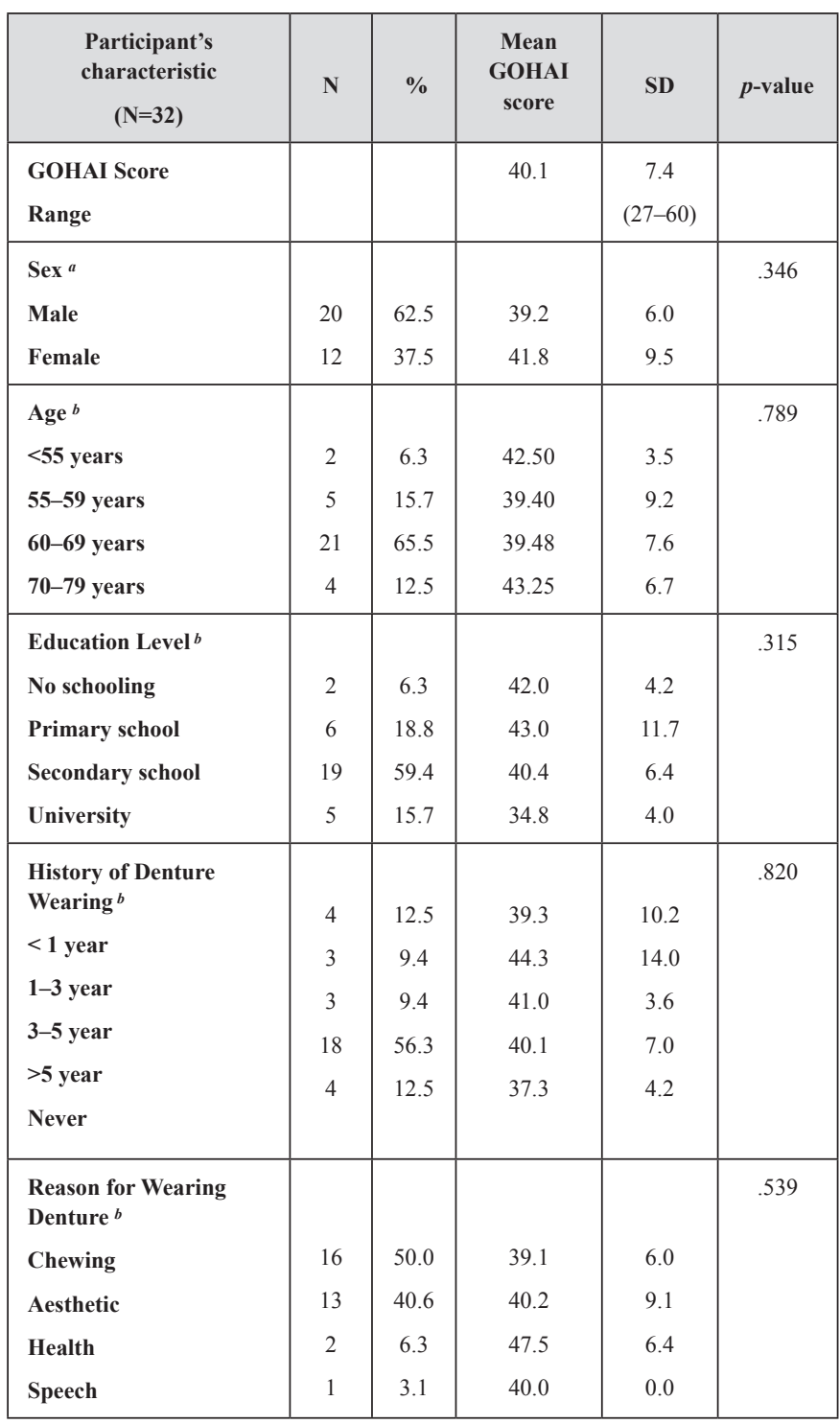

\section{Discussion}

TThis research was intended to evaluate the OHRQoL of the edentulous patients. There are many tools available to study the OHQoL, but the two most common measures used are GOHAI and OHIP-14. According to Ikebe et al (2012), GOHAI is more sensitive than OHIP-14, hence the choice of measures in this study. However, there was no measurements has proven that one measures is 
superior than the others [7]. Unfortunately, the relationship between gender, age, level of education, history of wearing denture and reason of wearing denture with the oral health related quality of life (OHRQoL) could not be established due to small sample size. For the improvement, larger sample size is needed in order to look for the significant association between those variables with the OHRQoL.

Table 3. Percentage of subjects responding 'sometimes,' 'often,' 'very often,' or 'always' to each GOHAI and mean each of the items.

\begin{tabular}{|c|c|c|}
\hline GOHAI ITEMS & $\begin{array}{l}\text { Percentage of } \\
\text { participants } \\
\text { responding positive } \\
\text { response }(\%)^{*}\end{array}$ & Mean (SD) \\
\hline \multicolumn{3}{|l|}{ Functional limitation } \\
\hline 2. Trouble biting and chewing & 64 & $1.66(1.6)$ \\
\hline 3. Able to swallow comfortably & 80 & $3.19(1.5)$ \\
\hline 4. Problem to speak clearly & 24 & $3.53(1.6)$ \\
\hline \multicolumn{3}{|l|}{ Pain and discomfort } \\
\hline 5. Able to eat without discomfort & 52 & $2.38(1.5)$ \\
\hline 8. Used medication to relieve pain & 16 & $4.56(0.8)$ \\
\hline 12. Sensitive to hot, cold or sweet foods & 20 & $4.31(1.1)$ \\
\hline \multicolumn{3}{|l|}{ Psychological impacts } \\
\hline 7. Pleased with the look of teeth & 56 & $2.25(1.4)$ \\
\hline 9. Worried about teeth, gums or dentures & 48 & $3.50(1.5)$ \\
\hline $\begin{array}{l}\text { 10. Self-conscious of teeth, gums or } \\
\text { dentures }\end{array}$ & 16 & $4.19(1.4)$ \\
\hline $\begin{array}{l}\text { 11. Uncomfortable eating in front of } \\
\text { others }\end{array}$ & 20 & $3.69(1.5)$ \\
\hline \multicolumn{3}{|l|}{ Behavioural impact } \\
\hline 1. Limit the kinds of food & 64 & $2.41(1.8)$ \\
\hline 6. Limit contact with people & 20 & $4.31(1.3)$ \\
\hline
\end{tabular}

In this present study, the most prevalent impact found in the functional limitation and behavioural domains with $64 \%$ of the participants had responded positive response in both negatively worded items (trouble biting and chewing as well as limit the kinds of food). On the other hand, pain and discomfort domain (used of medication to relieve pain) found as the least prevalent impact in OHRQoL. The research conducted by Adam (2006) using OHIPEDENT exhibited functional limitation as the most prevalent impact and psychological discomfort and physical pain came after as second and third most prevalent impact [8]. However, physical pain is the most prevalent impact according to Heydecke et al (2004) as the research used OHIP-DENT to measure OHRQoL of the samples [9].

In this study, the lowest mean for GOHAI item falls in functional limitation (trouble in biting and chewing) as it contributes a significance burden on individual as well as community. The complete denture patient experience more mastication problem compared to their dentate counterpart [8]. Meanwhile, an item in pain and discomfort domain which is "used of medication to relieve the pain" has highest mean. In other words, either the participant did not get bothered by pain while wearing denture or they were able to tolerate the pain. There was no standard classification on GOHAI score which indicates good or poor QoL. However, there was another classification used but it cannot be compare with this result because it use modified rating score. Hence, as the summary mean of GOHAI score is lower than 41 , it means that almost all participants $(68.7 \%)$ have relatively poor QoL and need treatment $[5,8]$.

Lastly, the main reason to seek complete denture treatment in this group of patients was to enhance chewing capability.

\section{Conclusion}

The QoL of geriatric patient was mostly affected by oral function and the least affected was seen in pain and discomfort. Thus, despite wearing complete denture, denture replacement is essential to improve Quality of life in geriatric patient.

\section{Acknowledgement}

This study was Funded by Short Term Grant Research Universiti Sains Islam Malaysia PPP/USG-0115/FPG/30/12715. We express our particular appreciation for the assistance of Azlan Jaafar, statistics advisor for this project.

\section{References}

1. Mojon P (2003) The world without teeth: demographic trends. Fastest clincal dentistry insight engine Pg No: 18-21.

2. Laurina L, Soboleva U (2006) Construction faults associated with complete denture wearers' complains. Stomatologija, Baltic Dental and Maxillofac J 8: 61-64. [crossref]

3. John MT, Feuerstahler L, Waller N, Baba K, Larsson P, et al. (2014) Confirmatory factor analysis of the Oral Health Impact Profile. J Oral Rehabil 41: 644-652. [crossref]

4. Atchison KA, Dolan TA (1990) Development of the Geriatric Oral Health Assessment Index. J Dent Educ 54: 680-687. [crossref]

5. Othman WN, et al. (2012) Validation of the Geriatric Oral Health Assessment Index (GOHAI) in the Malay language. Association between self-assessment of complete dentures and oral health-related quality of life. J Oral Rehabilitation 39: 847-857.

6. Ikebe K, Hazeyama T, Enoki K, Murai S, Okada T, Kagawa R, et al. (2012) Comparison of GOHAI and OHIP-14 measures in relation to objective values of oral function in elderly Japanese. Community dentistry and oral epidemiology 40: 406-414. [crossref]

7. Locker D, Matear D, Stephens M, Lawrence H, Payne B (2001) Comparison of the GOHAI and OHIP-14 as measures of the oral health-related quality of life of the elderly. Community Dent Oral Epidemiol 29: 373-381. [crossref]

8. Adam RZ (2006) Do complete dentures improve the quality of life of patients? (Doctoral dissertation, University of the Western Cape).

9. Heydecke G, Tedesco LA, Kowalski C, Inglehartet MR (2004) Complete dentures and oral health-related quality of life - do coping styles matter? Community Dent Oral Epidemiol 32: 297-306.

Citation:

Tine Martina Winarti, Norlela Yacob, Wan Nor Syariza Wan Ali, Aida Ali (2019) The Assessment of Quality of Life Using GOHAI Among Edentulous Patients. J Dent Maxillofacial Res Volume 2(1): 1-3. 